
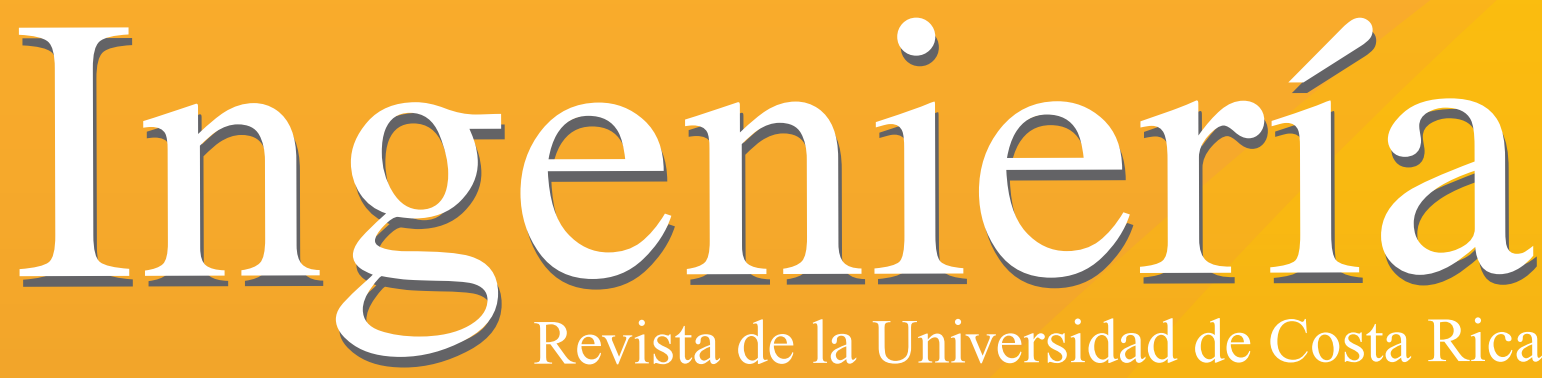

Revista de la Universidad de Costa Rica ENERO/ DICIEMBRE 2010 - VOLUMEN 20 - Número (1 y 2)
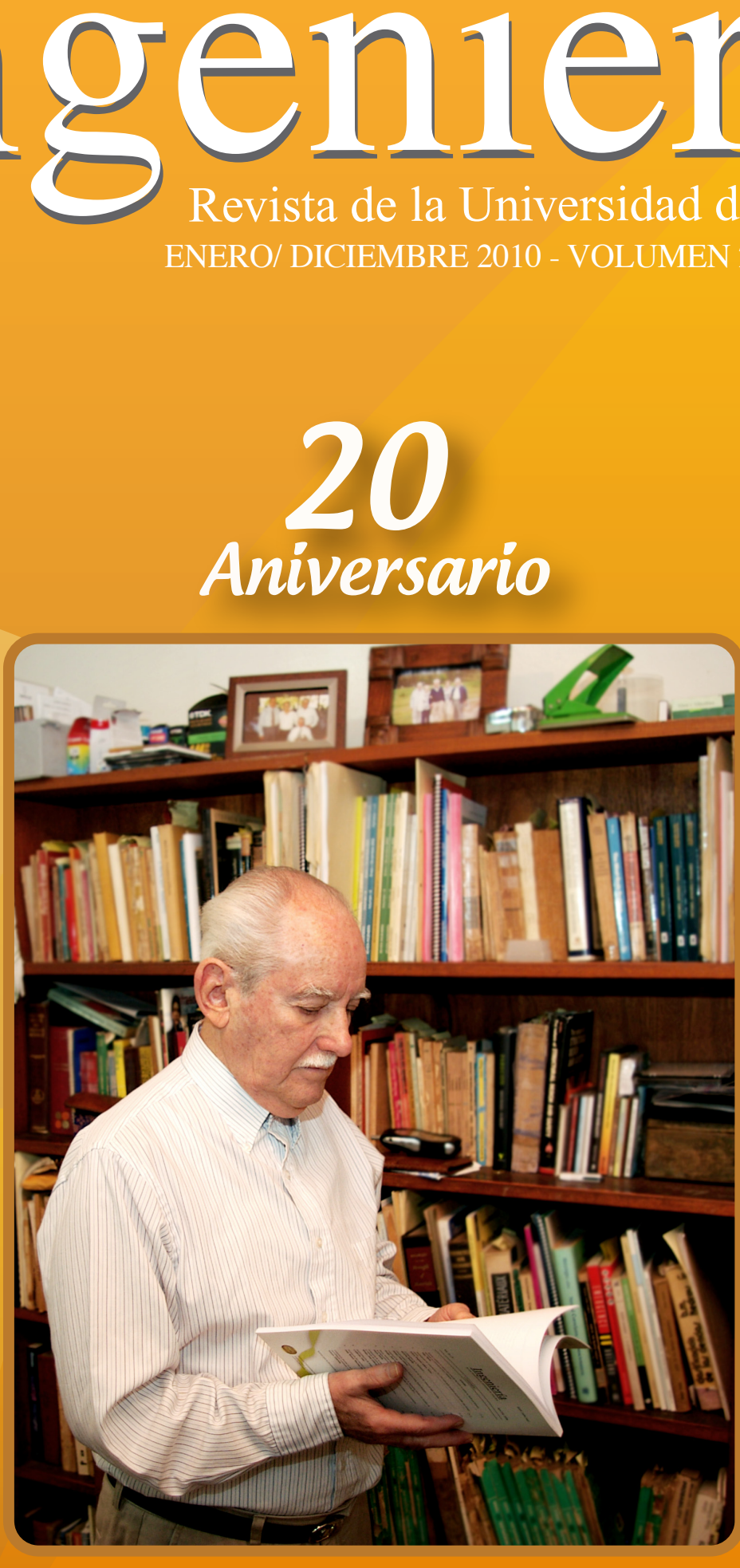

$\stackrel{\circ}{\circ}$

$\frac{\varrho}{\frac{0}{0}}$ 


\section{Análisis y comentarios}





\title{
MATERIALES ECOLÓGICOS: NI TAN FÁCILES DE INDENTIFICAR, NI TAN FÁCILES DE UTILIZAR!
}

\section{José Ali Porras Salazar Rafael Tenorio Monge}

\begin{abstract}
Palabras clave: materiales, materiales amigables al ambiente, materiales verdes (ecológicos), construcción, ecología, impacto medioambiental
\end{abstract}

Keywords: materials, green sustainable materials, environmental impact, construction.

Recibido: 10 de octubre del 2009 • Aprobado: 17 de noviembre del 2010

Hasta hace pocas décadas se tenía la impresión de que el planeta disponía de una cantidad de recursos naturales y energía, prácticamente inagotables. Además, en su vasta superficie y atmósfera el ser humano era capaz de emitir, casi sin límite, todo tipo de contaminantes sin que se dieran impactos significativos que la naturaleza sufriera cambios apreciables. Hoy se tiene constancia de que esto no es así y conforme el deterioro medioambiental se ha hecho más evidente y los científicos han encontrado un mayor número de relaciones causales entre las acciones antropogénicas y el cambio climático, se hace imprescindible que tanto los sectores productivos y económicos como el resto de la sociedad, realicen un cambio radical en sus patrones de producción y consumo.

A pesar de su importancia como uno de los mayores dinamizadores de la economía, el sector construcción es a su vez uno de los que más contribuye con el deterioro ambiental (Cuadro 1), ya que en sus fases de extracción y manufactura de materiales, edificación, mantenimiento y demolición, consume una gran cantidad de recursos no renovables y es una importante fuente de residuos y contaminación del aire, el agua y el suelo. Ejemplo de ello es que en la Unión Europea se estima que la construcción de edificios consume cerca del $40 \%$ de los materiales, genera el $40 \%$ de los residuos y requiere del $40 \%$ de la energía primaria (Baño \& Vigil-Escalera, 2005).

Por consiguiente, es urgente un cambio de mentalidad en todos los sectores ligados al proceso constructivo, que permita la adopción de decisiones encaminadas hacia la sostenibilidad. En este sentido uno de los aspectos que tiene mayor peso en el daño medioambiental son los materiales, de cuya correcta escogencia dependen en gran parte la minimización del impacto de procesos posteriores como el sistema constructivo, el consumo de agua y energía, los desechos, el mantenimiento y la demolición de los inmuebles, entre otros.

Históricamente, restricciones humanas y físicas como la topografía, la distancia y los

Cuadro 1. Incidencia ambiental de los materiales de construcción.

\author{
Consumo de energía \\ Consumo de recursos naturales \\ Impacto sobre los ecosistemas \\ Emisiones que generan \\ Comportamiento como residuo \\ Fuente: (Construmática, 2009).
}


límites administrativos (Rodrigue, Comtois \& Slack, 2009) así como la falta de sistemas de transporte avanzados, obligaban a la población a obtener los materiales para sus edificios en lugares aledaños a la construcción, los cuales en su mayoría eran simples y poco procesados, con lo que unido a una baja demanda, provocaban poca presión sobre el medio circundante.

No obstante, en la actualidad, con el aumento de la movilidad y la demanda de materiales por el crecimiento económico y demográfico, se ha pasado del uso de materias primas locales a un sistema centralizado de gran escala y con distribución global, donde la manufactura de los productos para la construcción conlleva un número cada vez mayor de procesos, algunos de los cuales dan como resultado una serie de compuestos en los cuales se suelen utilizar varios tipos de materiales unidos por aditivos químicos.

Esta situación ha motivado que gran cantidad de los impactos medioambientales por manufactura o extracción pasen desapercibidos en el sitio de la construcción, ya que estos son provocados a cientos e incluso miles de kilómetros. Por ejemplo, la tala de bosques en un área cercana a la obra puede ser demostrada fácilmente, pero es complicada de comprobar y aún más de interiorizar si esta se da como proceso previo a la extracción de aluminio o hierro en un territorio situado a gran distancia. Unido a ello, esta práctica hace casi imposible corroborar los procesos de producción que se dan en regiones lejanas -en caso de conocer la procedencia del material-, lo que en ocasiones da pie a que se pasen por alto leyes locales, especialmente cuando estos procedimientos se realizan en países con una jurisdicción ambiental laxa.

Uno de los aspectos que más preocupa de este sistema, es que por múltiples motivos se mantienen ocultos algunos de los costos por daños al medio ambiente y a la salud, conocidos como externalidades negativas. Este tipo de prácticas se dan, por ejemplo, en el transporte de los materiales, donde las lesiones provocadas por la emisión de gases o los accidentes no son compensados mediante un canon o impuesto a los usuarios. Unido a lo anterior, el modelo de producción centralizada y de distribución global no fomenta el desarrollo de la industria local y ha provocado que poco a poco se haya pasado de una mano de obra altamente calificada a una barata y de escaso conocimiento.

Como respuesta a esta problemática, el mercado ha puesto a disposición de los usuarios un grupo de materiales que se han denominado ecológicos, "verdes" o amigables con el ambiente. Sin embargo la selección y utilización de este tipo de productos es una de las áreas más confusas y controversiales del diseño con múltiples variables y gran cantidad de respuestas correctas e incorrectas (Calkins, 2009).

Las contrariedades comienzan con la propia definición de "ecológico" yaqueexisten diferencias entre los expertos sobre las implicaciones que tiene el uso de esta palabra como un adjetivo. Aun así se le podría dar este calificativo a aquellos materiales en cuyo proceso de extracción y/o fabricación se minimicen los impactos negativos sobre el medio ambiente, pero además que estén diseñados de forma tal que potencien esta reducción en las restantes etapas del ciclo de vida del edificio: transporte de materiales, construcción, funcionamiento y demolición (Cuadro 2).

No obstante existe una importancia relativa en la magnitud y los riesgos del impacto medioambiental, la cual va a depender en parte de las prioridades que defina el comprador o el comercializador a la hora de seleccionar los productos. Por ejemplo, el cemento Portland podría considerarse ambientalmente amigable si los aspectos por evaluar son la durabilidad y el uso de materiales producidos regionalmente, pero estaría fuera de la lista si lo que se considera es el calentamiento global o productos de alto consumo energético (Calkins, 2009). Siendo así, es importante estar seguro del valor que se le asignará a cada uno de los indicadores y si estos tienen concordancia con la realidad económica, ambiental y social del sitio donde se realiza la edificación.

A raíz de la dificultad que conlleva la valoración y la ponderación de variables tan divergentes, es que se han desarrollado metodologías de evaluación cuya finalidad es analizar de forma objetiva, metódica y científica, el impacto originado por un producto mediante el uso de indicadores preestablecidos. Entre las más conocidas se encuentran la Evaluación de la Sostenibilidad (SA), la Evaluación de la Energía Incorporada (EE) y el Análisis de Ciclo de $\operatorname{Vida}^{2}$ (LCA) (Calkins, 2009). 
Cuadro 2. Características de los materiales sostenibles.

\author{
Proceden de fuentes renovables y abundantes \\ No contaminan \\ Consumen poca energía en su ciclo de vida \\ Son duraderos \\ Pueden estandarizarse \\ Son fácilmente valorizables \\ Proceden de producción justa \\ Tienen valor cultural en su entorno \\ Tienen bajo costo económico
}

Fuente: (Baño y Vigil-Escalera, 2005).

Esta última creada en 1990 fue acogida por la Organización Internacional para la Estandarización (ISO por sus siglas en inglés) la cual en 1993 conformó el Comité Técnico que desarrolló la normativa internacional en materia de gestión ambiental, plasmada en la serie de normas ISO 14040. Su accionar está comprendido de cuatro fases:

1. Metas y alcances del estudio.

2. Inventario de las entradas y salidas relevantes del sistema del producto.

3. Evaluación de los potenciales impactos medioambientales asociados con las entradas y salidas identificadas en el inventario.

4. Interpretación de los resultados de las fases de análisis de inventario y evaluación de impacto de acuerdo con los objetivos del estudio.

Sin embargo, aun cuando el (Análisis del Cliclo de Vida) tiene la reputación de ser una de las herramientas más completas para la evaluación de impactos sobre el ambiente y la salud humana de materiales y productos, es a su vez un instrumento complejo, que requiere de gran experiencia para establecer el peso de cada uno de los impactos y consume una gran cantidad de tiempo, por lo que se podría considerar fuera del alcance de la mayoría de diseñadores y por consiguiente, utilizada principalmente por analistas profesionales.

En la actualidad existen en el mercado varias herramientas de cálculo basadas en el Análisis de Ciclo de Vida, como el Athena Evironmental Impact Estimator desarrollado por el Instituto Athena de Materiales Sostenibles de Canadá, o el Building for Environmental and Economic Sustainability (BEES) del Instituto Nacional de Estandarización y Tecnología (Calkins, 2009).

Paralelamente, coexisten algunos otros métodos que al valerse de un único indicador son más sencillos. Uno de los más utilizados es la medición del consumo de energía empleado en la producción de un kilogramo o metro cúbico de un material determinado. A pesar de ser un cálculo que se lleva a cabo relativamente rápido, lamentablemente causa algunas confusiones ya que no toma en cuenta varios factores determinantes. Ejemplo de lo anterior es que para fabricar un metro cúbico de acero se necesitaría sesenta y cinco veces más energía que para producir la misma cantidad de concreto. Aun así, si se toma en cuenta la resistencia, una viga de acero producida con tan solo un $25 \%$ más de energía, podría soportar lo mismo que una de concreto, con las ventajas de ser reutilizable y reciclable.

Otro inconveniente que se presenta con los materiales amigables con el medio ambiente es que un gran número de ellos son naturales ${ }^{3}$, lo que dificulta su uso en la construcción. Esto se debe a razones como la disponibilidad a mediana y gran escala, las dificultades que se presentan en la instalación y el mantenimiento o la falta de mano de obra especializada y de procesos de certificación que garanticen estándares de calidad, durabilidad o resistencia. Incluso existen casos en 
donde este tipo de inconvenientes puede causar un diferencial de costos tan alto, que el proyecto deja de ser económicamente rentable y su uso se desecha.

Por lo tanto, antes de tomar la decisión de cuáles materiales utilizar es importante conocer si los que se promocionan como ecológicos realmente lo son e informarse, aunque sea de forma general, sobre las magnitudes y los riesgos del impacto que producen. Además se debe tener en cuenta que no siempre la respuesta correcta está en el empleo de la última tecnología en materiales "verdes"; quizás la solución en algunos casos se halle más bien en la utilización de productos convencionales de manera distinta. En este sentido se podría pensar en el uso de agregados reciclados para la sustitución y compactación del suelo o el diseño de un sistema estructural que permita el reemplazo de piezas o un fácil desensamble.

Siendo así, no debería parecer extraño que la paleta de materiales utilizada en un edificio contenga unos productos más ecológicos que otros, ya que también se deben considerar otros aspectos como el diseño, el clima, el sistema constructivo y hasta el mismo instalador, lo cual lleva a tener en una misma edificación lo que algunos expertos llaman una gama de verdes ${ }^{4}$.

Finalmente, las dificultades encontradas a la hora de elegir materiales ecológicos no debería ser un desincentivo para su uso en el país, sino más bien un estímulo para que se elaboren sistemas nacionales de certificación de productos a costos razonables -o adaptar algunos de los internacionales-, que permitan a las empresas medir la magnitud y riesgos del impacto de los materiales que producen y/o importan de forma sistemática y transparente, trasladando esta información al consumidor para que este pueda tomar una elección apropiada con conocimiento de causa.

\section{NOTAS}

1. La base de este artículo forma parte de una consultoría desarrollada por los autores para el Banco Interamericano de Desarrollo (BID) y el Sistema Nacional de Áreas de Conservación (SINAC).

2. Las siglas corresponden a los nombres en inglés: Sustainability Assesment (SA) Embodied Energy (EE) y Life-Cycle Assesment o Life-
Cycle Analisys (LCA).

3. Materiales ofrecidos por la naturaleza y que son utilizados en la construcción, sin sufrir complejos procesos de industrialización.

4. Traducido por el autor de: Shades of green.

\section{REFERENCIAS BIBLIOGRÁFICAS}

Baño, A. \& Vigil-Escalera, A. (2005). Guía de construcción sostenible. España: Instituto Sindical de Ambiente, Trabajo y Salud.

Calkins, M. (2009). Materials for sustainable sites. Haboken, N. J.: John Wiley \& Sons.

Materiales de construcción sostenibles. Extraído el 7 se septiembre del 2009 de http://www. construmatica.com/construpedia/Materiales_ de_Construcci\%C3\%B3n_Sostenibles

Rodrigue, J. P., Comtois, C. \& Slack, B. (2009). The geography of transport systems $\left(2^{\mathrm{a}} \mathrm{ed}.\right)$. New York: Routlege.

\section{SOBRE LOS AUTORES}

\section{José Alí Porras Salazar}

Arquitecto de la Universidad de Costa Rica

M. Sc. en Medio Ambiente y Arquitectura Bioclimática de la Universidad Politécnica de Madrid, España

Especialización en Desarrollo Sostenible del INCAE Especialización en Políticas Energéticas de la Universidad Técnica de Berlín e InWent

Labora para la empresa Ecobalance Consultores

Profesor de la Escuela de Arquitectura, de la Universidad de Costa Rica

Investigador del Instituto de Investigaciones de Ingeniería, de la Universidad de Costa Rica

Teléfono: (506) 8305-0666

Correo electrónico: joseali@live.com

\section{Rafael Tenorio Monge}

Arquitecto de la Universidad de Costa Rica

Consultor de firmas de diseño como Entrópica CR, TAG Arquitectos y Ecobalance Consultores 